\title{
Nutritional management during treatment for head and neck cancer
}

\author{
Christina Wagner (D)
}

Received: 2 March 2020 / Accepted: 23 April 2020 / Published online: 14 May 2020

(C) The Author(s) 2020

\begin{abstract}
Summary
Background The majority of patients who suffer from head and neck cancer are malnourished even prior to treatment initiation. In addition, side effects from cancer therapy including change in taste, mucositis, nausea or diarrhea increase patients' malnutrition. Therefore, early management is crucial to improve nutritional status, prognosis and quality of life of patients.

Methods A literature research was performed in PubMed, Medline and other available databases. The guidelines of the German Society for Nutritional Medicine, the European Society for Clinical Nutrition and Metabolism and common recommendations of other countries were selected, analyzed and summarized.

Results Early screening for malnutrition is recommended for all cancer patients. Adequate intake of energy and protein should be ensured which may be achieved by consumption of oral nutritional supplements or enteral nutrition such as tube feeding.

Conclusion It is important to determine the best course of management to maintain body weight and reduce typical adverse effects of malnutrition to improve quality of life. All patients at any stage of their treatment should receive intensive dietary counseling.
\end{abstract}

Keywords Nutrition · Oncological dietetics · Dietary deficiency · Malnutrition · Cachexia · Head and neck oncology

\section{Wagner, MSc $(\triangle)$}

Department of Medicine I, Division of Oncology, Medical University of Vienna, Währinger Gürtel 18-20, 1090 Vienna, Austria

christina.wagner@meduniwien.ac.at
Head and neck cancer is the sixth most common cancer worldwide. Annually 1200 new cases are diagnosed in Austria [1, 2]. Up to $60 \%$ of patients being treated for locally advanced head and neck squamous cell cancer suffer from malnutrition prior to even starting treatment [3]. Unintentional weight loss is a result of reduced food intake, systemic inflammation and persistent catabolism [4]. Malnutrition with a weight loss up to $10 \%$ of baseline body mass often leads to more adverse effects, reduced prognosis and reduced quality of life of patients. Therefore, early management is essential to improve nutritional status, patient outcome and quality of life [4].

\section{Adverse effects of malnutrition}

Cancer patients are at risk of malnutrition due to disease progress and antitumor therapy. The risk for patients with head and neck cancer is even higher due to the location of their tumor. The development of difficulties in chewing and dysphagia is increased [5].

Chemotherapy, radiotherapy and surgery mostly affect nutritional intake and may cause side effects. The most common adverse events in head and neck cancer after initiation of treatment are mucositis, xerostomy, changes in taste, pain, nausea and vomiting. As a result, they lead to reduced food intake [5].

Lifestyle factors such as consumption of alcohol and tobacco use also contribute to malnutrition. Alcohol provides empty calories and suppresses appetite. Alshadwi et al. [3] have indicated that head and neck cancer patients with a history of alcohol misuse have a much lower intake of fresh fruits and vegetables. Thus, the probability of malnutrition is increase in this population group compared to other tumor entities [3].

A weight loss of more than $10 \%$ of the baseline body mass may lead to various adverse effects such 
as an increased risk of infection, delayed wound healing, muscle waste, depression and a reduced response to chemo- or radiotherapy [4]. Unintentional weight loss may lead to cachexia which in turn results in decreased appetite, fat and muscle loss and metabolic alterations [5].

\section{Nutritional assessment}

Nutritional screening at the time of diagnosis is an essential aim to identify patients who are at risk of becoming malnourished. Several screening tools exist to detect those who require early nutritional counseling $[4,5]$, for example, the Nutrition Risk Screening 2002 (NRS-2002) which was developed for inpatients to predict postoperative complications and the length of hospitalization.

The Malnutrition Universal Screening Tool (MUST) - a five-step screening instrument to identify malnourished outpatients [4] - measures height, weight, $\mathrm{BMI}$ and unintentional weight loss over a period of 6 months. A high MUST score results in an elevated risk of malnutrition. The MUST was developed by the British Association for Parenteral and Enteral Nutrition who provides management guidelines for patients at medium or high risk of malnutrition [6].

The Subjective Global Assessment (SGA) is one of the first validated screening tools often used in clinical trials and therefore at present it is the most used assessment tool to evaluate weight history, physical examination, muscle wasting, fat loss and gastrointestinal symptoms. Nutritional status is assessed by a clinical member through anthropometric measurements. Measurements of waist, chest and arms are performed to assess body fat mass and muscle mass including muscle strength [7]. Similar to the SGA is the Patient Generated Subjective Global Assessment (PG-SGA). The PG-SGA includes an additional questionnaire to be completed by the patients themselves $[3,4]$.

However, the most frequently used methods of assessing nutritional status are evaluation of patients' weight history and physical examination in the clinic [5]. Body mass index (BMI) has been found to be a useful measurement that sets body weight in relation to height. The BMI estimates the normal body weight. A BMI under $18.5 \mathrm{~kg} / \mathrm{m}^{2}$ is considered as underweight [3, 8].

Nutritional assessment should be repeated weekly. Weight should be recorded every time a patient visits the hospital. Patients with a weight loss of $2 \mathrm{~kg}$ or more within a 2-week period are required to adapt nutritional counseling. According to the guidelines of ESPEN and DGEM, nutritional screening should be repeated at least every 8 weeks $[4,9]$. The majority of patients have difficulties in implementation of the planned nutritional counseling due to adverse events [10]. In order to avoid long-term consequences pa- tients should be followed up at least 6 months posttreatment by nutritional experts [11].

Further factors to be protocolled frequently are the ability to chew and swallow, changes in appetite and gastrointestinal functions. Furthermore, daily medication, general physical examination and blood chemistry parameters should be included in the nutritional assessment $[4,9]$.

\section{Treatment of malnutrition}

Nutritional support is recommended for all cancer patients at any stage of their treatment. Nutritional treatment prevents both disease- and treatment-related weight loss. The main aim is to improve patients' quality of life to reduce therapy-related adverse events and to minimize side effects due to malnutrition. For nutrition counseling three methods are used in clinical practice: oral, enteral and parenteral nutrition [4, 9].

\section{Oral nutrition}

Until now no prospective studies have been conducted for the optional energy and nutrition intake for cancer patients. Nutritional requirements are based on general recommendations. Patient should be encouraged to eat a balanced diet to meet the nutritional requirements. The main emphasis for mild malnutrition should be on a high caloric and high protein diet $[3,11]$. According to the ESPEN guidelines of nutrition the energy intake ranges from 25 to $30 \mathrm{kcal} / \mathrm{kg} / \mathrm{day}$. Protein supply is recommended with 1.2 to $1.5 \mathrm{~g} / \mathrm{kg} /$ day. In cases of systematic inflammation the protein intake can be adapted to $2 \mathrm{~g} / \mathrm{kg} /$ day, however only in patients with normal kidney function $[4,9]$.

Primary advice for increased caloric intake is to use whole dairy products instead of fat-free alternatives or mayonnaise instead of dressing, butter to cook instead of oils or crème soup instead of bouillon. However, this is often difficult and additional intake of supplements is required. For patients with mild or moderate malnutrition, it can be helpful to start oral nutrition support. To achieve a high caloric-protein diet oral nutritional supplementation (ONS) can be started at any point from diagnosis $[4,11]$. ONS are enteral formulas which provide necessary macro- and micronutrients. They are commercially available, can be used as a simple drink or to enrich traditional fare $[3,4]$. For patients with dysphagia, ONS can improve oral intake due to the soft texture [12].

\section{Enteral nutrition}

Enteral nutrition is recommended for subjects who are unable to meet their nutrition requirements through oral diet despite nutritional advice and ONS. If a patient eats inadequately or has not been eating 
for more than one week, artificial nutrition is indicated. Arends et al. recommend enteral nutrition if food intake is less than $60 \%$ of the individual requirement for more than 2 weeks [4, 9]. In general, patients are at high risk for malnutrition [3]. Clinical consideration for enteral nutrition should include the location of tumor, the actual treatment plan and the predicted duration of enteral feeding. Various types of feeding tubes are available. If a patient only needs a short-term use of enteral feeding, the recommended tubes are nasogastric or nasojejunal tubes. These kinds of tubes can be used less than 4 weeks. Enteral feeding with gastrostomy, gastrojejunostomy and jejunostomy tubes is recommended for longterm use [11].

Alshadwi et al. describe that gastrostomy tubes should be used as prophylaxis before radiotherapy to prevent weight loss, treatment interruption and dehydration. Evidence from clinical studies suggests that starting early enteral feedings prior to major surgery is an advantage, especially for improved wound healing [3]. Early pre- and postoperative enteral nutrition was shown to significantly reduce the length of hospitalization in patients with severe malnutrition [10]. Arends et al. specify that percutaneous endoscopic gastrostomies are to be preferred to nasogastric feeding $[4,9]$.

\section{Parenteral nutrition}

Parenteral nutrition is rarely used in cancer patients and should be consider as last feeding choice due to increased complications including infections, sepsis and metabolic derangements. Parenteral nutrition is only considered if enteral nutrition is insufficient or contraindicated [3]. For patients who need immediate metabolic treatment and suffer from severe intestinal insufficiency, total parenteral nutrition is recommended $[4,9]$.

\section{Conclusion}

Numerous side effects of anticancer treatment like changes in taste, mucositis, nausea or diarrhea enhance malnutrition and have a significant negative impact on mortality, morbidity and quality of life. It is important to maintain body weight and nutritional status throughout oncologic treatment. Most common methods for this are the evaluation of weight history, physical examination and measurement of BMI.

Nutritional needs of each patient are often very complex and have to be adapted during the course of cancer therapy. Oral nutrition should be encouraged at all times. However, administration of oral nutritional supplements may be required if oral feeding is inadequate to maintain body weight.

Enteral and parenteral nutrition via tube feeding should be considered for further weight loss.
It is important to determine the best course of nutrition intake to avoid malnutrition. All patients at any stage of their treatment should receive intensive dietary counseling.

\section{Take home message}

- Early nutritional screening is important for patients at high risk of malnutrition.

- Nutritional management should be provided to each patient at any stage of their treatment.

- Oral or enteral nutrition should be initiated as early as required.

- Good nutritional status improves quality of life, patient outcome and survival.

- Dieticians should be an important part in healthcare team during oncological treatment and rehabilitation.

Funding Open access funding provided by Medical University of Vienna.

Conflict of interest C. Wagner declares that she has no competing interests.

Open Access This article is licensed under a Creative Commons Attribution 4.0 International License, which permits use, sharing, adaptation, distribution and reproduction in any medium or format, as long as you give appropriate credit to the original author(s) and the source, provide a link to the Creative Commons licence, and indicate if changes were made. The images or other third party material in this article are included in the article's Creative Commons licence, unless indicated otherwise in a credit line to the material. If material is not included in the article's Creative Commons licence and your intended use is not permitted by statutory regulation or exceeds the permitted use, you will need to obtain permission directly from the copyright holder. To view a copy of this licence, visit http://creativecommons.org/licenses/by/4.0/.

\section{References}

1. StatistikAustria. Kopf, Hals. 2020. https:/ /www.statistik.at/ web_de/statistiken/menschen_und_gesellschaft/ gesundheit/krebserkrankungen/kopf_hals/index.html. Accessed 29 Jan 2020.

2. World Health Organization. Locally advanced squamous carcinoma of the head and neck. 2020. https:// www.who.int/selection_medicines/committees/expert/ 20/applications/HeadNeck.pdf. Accessed 29 Jan 2020.

3. Alshadwi A, Nadershah M, Carlson E, et al. Nutritional consideration for head and neck cancer patients: a review of the literature. J Oral Maxillofac Surg. 2013;71:1853-60. https://doi.org/10.1016/j.joms.2013.04.028.

4. Arends J, Bertz H, Bischoff SC, et al. Klinische Ernährung in der Onkologie. Aktuel Ernahrungsmed. 2015;40:e1-e74. https://doi.org/10.1055/s-0035-1552741.

5. Sandmael JA, Sand K, Bye A, et al. Nutritional experiences in head and neck cancer patients. Eur J Cancer Care. 2019;28(6):e13168. https://doi.org/10.1111/ecc.13168.

6. BAPEN. Introducing 'MUST'. 2016. https://www.bapen. org.uk/screening-and-must/must/introducing-must. Accessed 5 Feb 2020.

7. DeutscheGesellschaftfürErnährungsmedizine. V.. Screening. 2020. http://dgem.de/screening. Accessed5 Feb 2020.

8. Elmadfa I. Ernährungslehre. Stuttgart: Eugen Ulmer; 2004. 


\section{short review}

9. Arends J, Bachmann P, Baracos V, et al. ESPEN guidelines on nutrition in cancer patients. Clin Nutr. 2017;36:11-48. https://doi.org/10.1016/j.clnu.2016.07.015.

10. Orell H, Schwab U, Saarilahti K, et al. Nutritional counseling for head and neck cancer patients undergoing (chemo)radiotherapy-A prospective randomized trial. Front Nutr. 2019; https:// doi.org/10.3389/fnut2019.00022.

11. Talwar B, Donnelly R, Skelly R, et al. Nutritional management in head and neck cancer: United Kingdom National Multidisciplinary Guidelines. J Laryngol Otol. 2016;130(S2):S32-S40. https://doi.org/10.1017/ s0022215116000402.

12. Kristensen MB, Isenring E, Brown B. Nutrition and swallowing therapy strategies for patients with head and neck cancer. Nutrition. 2020;69:110548. https://doi.org/10. 1016/j.nut.2019.06.028.
Publisher's Note Springer Nature remains neutral with regard to jurisdictional claims in published maps and institutional affiliations.

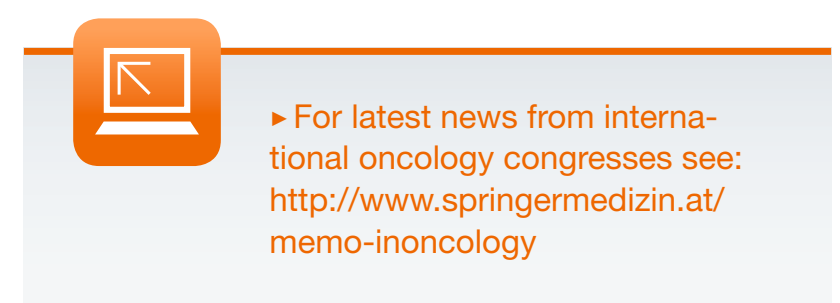

